\title{
Statistical mechanics of double-stranded semi-flexible polymers
}

\author{
Tanniemola B. Liverpool ${ }^{\dagger}$, Ramin Golestanian ${ }^{\S}$ and Kurt Kremer $^{\dagger}$ \\ ${ }^{\dagger}$ Max-Planck-Institut für Polymerforschung, D-55021 Mainz, Germany \\ $\S$ Institute for Advanced Studies in Basic Sciences, Zanjan 45195-159, Iran
}

(September 18, 2018)

\begin{abstract}
We study the statistical mechanics of double-stranded semi-flexible polymers using both analytical techniques and simulation. We find a transition at some finite temperature, from a type of short range order to a fundamentally different sort of short range order. In the high temperature regime, the 2-point correlation functions of the object are identical to worm-like chains, while at low temperatures they are different due to a twist structure. In the low temperature phase, the polymers develop a kink-rod structure which could clarify some recent puzzling experiments on actin.
\end{abstract}

$87.15 \mathrm{By}, 36.20 \mathrm{Ey}, 61.25 \mathrm{Hq}$

A double-stranded semi-flexible polymer chain is the basic structure of many biopolymers of which examples are DNA and proteins such as actin. There has been a lot of recent experimental interest in systems of biopolymers ranging from the elasticty of biopolymer networks and its use in the prediction of the mechanical properties of cells to the direct visualisation of single chain properties. The model most used in the study of biopolymers is that of the worm-like chain [1] in which the polymer flexibility (structure) is determined by a single length, the persistence length $\ell_{p}$ which measures the tangent-tangent correlations. For example, DNA has a persistence length $\ell_{p} \approx 50 \mathrm{~nm}$ whilst for actin $\ell_{p} \approx 17 \mu \mathrm{m}$. These biopolymers are known to have a more complex 'twisted' structure. The multi-stranded nature of these polymers is also not taken into account in a simple worm-like chain model. It is not clear if such a fine structure will have an effect on the global properties of these objects. A possible effect of such fine structure is what we attempt to study in this Letter. Our model is, in a sense, microscopic because the interaction between the bend and twist degrees of freedom is a result. This is fundamentally diffent from previous approaches [2] which try to include the twist degrees of freedom by adding extra terms to the free energy.

We study a version of the rail-way track model of Everaers-Bundschuh-Kremer (EBK) [3] for a doublestranded semi-flexible polymer, embedded in a $d$ dimensional space for arbitrary $d$. Excluded volume and electrostatic interactions have been ignored. We find that the system, has qualitatively different properties in the low temperature and high temperature regimes, in contrast to what one might naively expect from an inherently one-dimensional system with local interactions and constraints. The tangent-tangent correlation function decays exponentially in the whole range of temperatures with a "tangent-persistence length" $\tilde{\ell}_{\mathrm{TP}}$ that has a very slow temperature dependence, and whose scale is determined by the (bare) persistence length of a single strand $\ell_{p}=\kappa / k_{B} T$ ( $\kappa$ is the bending stiffness of a single strand $)$.
Note that it is independent of $a$, the separation of the two strands, which is the other relevant length scale in the problem. However, the correlation function of the bond-director field, defined as a vector that determines the separation and coupling of the two strands of the combined polymer system, has different behaviour below and above the temperature $T_{c} \simeq 4.27 \kappa / d k_{B} a$. While it decays purely exponentially for $T>T_{c}$, there are additional oscillatory modulations for $T<T_{c}$. The related "bond-persistence length" $\tilde{\ell}_{\mathrm{BP}}$ does not change appreciably at high temperatures, where its scale is again set by $\ell_{p}$ alone. In the low temperature phase, however, $\tilde{\ell}_{\mathrm{BP}}$ does show a temperature dependence. In particular, $\tilde{\ell}_{\mathrm{BP}} \sim \ell_{p}^{1 / 3} a^{2 / 3} \propto T^{-1 / 3}$ for $T \rightarrow 0$, while $\tilde{\ell}_{\mathrm{BP}} \sim \ell_{p}$ for $T \sim T_{c}$. Similarly, the "pitch" $H$, defined as the period of oscillations in the low temperature regime, changes drastically with temperature, ranging from $H \sim \tilde{\ell}_{\mathrm{BP}} \sim \ell_{p}^{1 / 3} a^{2 / 3}$ near $T=0$, to $H \sim 0$ near $T=T_{c}$. At $T=0$ we regain a flat ribbon which has true long-range order in both the tangent and bond-director fields. The ribbon is essentially a rigid rod. As we approach $T=0$, the persistence lengths and the pitch diverge with the scaling $H \sim \tilde{\ell}_{\mathrm{BP}} \sim \tilde{\ell}_{\mathrm{TP}}^{1 / 3}$.

The system is composed of two semi-flexible chains, each with rigidity $\kappa$, whose embeddings in $d$-dimensional space are defined by $\mathbf{r}_{1}(s)$ and $\mathbf{r}_{2}(s)$. The Hamiltonian of the system can be written as the sum of the Hamiltonians of two worm-like chains subject to the inextensiblity constraints 洶. The ribbon structure is enforced by having $\mathbf{r}_{2}\left(s^{\prime}\right)$ separated from $\mathbf{r}_{1}(s)$ by a distance $a$, i.e. $\mathbf{r}_{2}\left(s^{\prime}\right)=\mathbf{r}_{1}(s)+a \mathbf{n}(s)$ where $\left|s-s^{\prime}\right|$ can be non-zero but is small. We have defined a bond-director field $\mathbf{n}(s)$, a unit vector perpendicular to both strands. The chains are assumed to have "permanent" bonds (e.g. hydrogen bonds) that are strong enough to keep the distance between the two strands constant. In Ref. [3], it is argued that the relevant constraint on the system would then be that in a bent configuration the arc-length mismatch between the two strands should be very small. We can calculate the arc-length mismatch for the bent configu- 
ration as $\Delta s=\left|\mathbf{r}_{2}(s)-\mathbf{r}_{1}(s)+a \mathbf{n}(s)\right|$, where $a$ is the separation of the strands. We impose the constraint as a hard one, namely, we set $\Delta s=0$, as opposed to Ref. [3]. Physically this means we do not allow bends in the plane of the ribbon. These bends are less important in $d>2$ because as we shall see the lower length-scale will be set by the 'pitch' which will make the in plane fluctuations of the ribbon irrelevant [3]. This simplifying assumption should not change the behavior of the system [5]. We implement the constraint $\Delta s=0$ by introducing the "midcurve" $\mathbf{r}(s): \mathbf{r}_{1}(s)=\mathbf{r}(s)+\frac{a}{2} \mathbf{n}, \quad \mathbf{r}_{2}(s)=\mathbf{r}(s)-\frac{a}{2} \mathbf{n}$. In terms of the tangent to the midcurve $\mathbf{t}=\mathrm{d} \mathbf{r} / \mathrm{d} s$ and the bond-director $\mathbf{n}$ the Hamiltonian of the system can now be written as

$$
\mathcal{H}=\frac{\kappa}{2} \int \mathrm{d} s\left[2\left(\frac{\mathrm{d} \mathbf{t}(s)}{\mathrm{d} s}\right)^{2}+\frac{a^{2}}{2}\left(\frac{\mathrm{d}^{2} \mathbf{n}(s)}{\mathrm{d} s^{2}}\right)^{2}\right]
$$

subject to the exact (local) constraints

$$
\left(\mathbf{t} \pm \frac{a}{2} \frac{\mathrm{d} \mathbf{n}}{\mathrm{d} s}\right)^{2}=1, \quad \mathbf{n}^{2}=1, \quad\left(\mathbf{t} \pm \frac{a}{2} \frac{\mathrm{d} \mathbf{n}}{\mathrm{d} s}\right) \cdot \mathbf{n}=0 .
$$

This completes the formulation of the model.

The statistical mechanics of semi-flexible chains are difficult due to the constraint of in-extensibility. Various approximation methods have been devised to tackle the problem. A successful scheme, that somehow manages to capture the crucial features of the problem, is to impose global (average) constraints rather than local (exact) ones [-14. This approximation is known to be good for calculating the average end-to-end length but not so good for the whole distribution. It corresponds to a saddle-point evaluation of the integrals over the Lagrange multipliers, that are introduced to implement the constraints [4]. In this sense, it is known to be a "meanfield" approximation in spirit. To study the effects of fluctuations on the mean-field result, we have performed a $1 / d$-expansion similar to the one successfully used by David and Guitter to study the crumpling transition of crystalline membranes [6]. We see that no divergent behaviour appears in the diagrams of the 2-point correlation functions which means that the mean-field behavior of these functions is not changed by fluctuations. This does not preclude differences in higher order correlation functions.

With the above discussion as justification, we apply the same approximation scheme to our problem defined above. The local constraints in Eq.(2) are relaxed to global ones. To do this, we add the corresponding "mass terms" to our Hamiltonian
$\frac{\mathcal{H}_{m}}{k_{B} T}=\int \mathrm{d} s\left[\frac{b}{\ell_{p}}\left(\mathbf{t}-\frac{a}{2} \frac{\mathrm{d} \mathbf{n}}{\mathrm{d} s}\right)^{2}+\frac{b}{\ell_{p}}\left(\mathbf{t}+\frac{a}{2} \frac{\mathrm{d} \mathbf{n}}{\mathrm{d} s}\right)^{2}+\frac{c a^{2}}{4 \ell_{p}^{3}} \mathbf{n}^{2}\right.$ $\left.+\frac{e}{\ell_{p}}\left(\mathbf{t}-\frac{a}{2} \frac{\mathrm{d} \mathbf{n}}{\mathrm{d} s}\right) \cdot \mathbf{n}+\frac{e}{\ell_{p}}\left(\mathbf{t}+\frac{a}{2} \frac{\mathrm{d} \mathbf{n}}{\mathrm{d} s}\right) \cdot \mathbf{n}\right]$, where $b, c$, and $e$ are dimensionless constants. We then determine the constants self consistently by demanding the constraints of Eq.(2) to hold on average, where the thermal average is calculated by using the total Hamiltonian $\mathcal{H}+\mathcal{H}_{m}$. Note that in choosing the above form, we have implemented the "label symmetry" of the chains, namely, that there is no difference between two chains. The self-consistency lead to the following set of equations for the constants $b$ and $c: \frac{1}{4 \sqrt{2 b}}+\frac{a^{2} \sqrt{c}}{4 d \ell_{p}^{2}}=\frac{1}{d}, c(b+\sqrt{c})=\frac{d^{2} \ell_{p}^{4}}{2 a^{4}}$, and $e=0$. The above equations, which are nonlinear and difficult to solve exactly, determine the behavior of $b$ and $c$ as a function of $u=a / \ell_{p}$. We have solved them numerically in $d=3$ and the solutions are given in Fig. 1. One can solve the equations analytically in two limiting cases. For $u \ll 1$ we find $b=d^{2} / 32$, and $c=(d / \sqrt{2})^{4 / 3} u^{-8 / 3}$, whereas for $u \gg 1$ we find $b=d^{2} / 8$, and $c=4 / u^{4}$. In Fig. 1, the behavior of $b$ and $c$ is plotted as a function of $u$. Note that $u$ is proportional to $T$ and can be viewed as a measure of temperature.

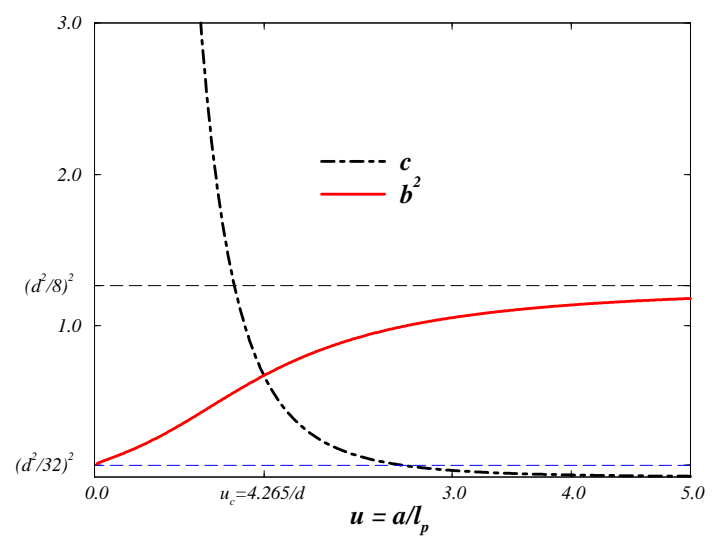

FIG. 1. The solution of the self consistent equations for the constants $b$ and $c$ as a function of $u=a / \ell_{p}$ in $d=3$. The value $u_{c} \simeq 4.27 / d$ corresponds to the transition point.

We can then calculate the correlation functions. For the tangent-tangent correlation one obtains

$$
\langle\mathbf{t}(s) \cdot \mathbf{t}(0)\rangle=\frac{d}{4 \sqrt{2 b}} \exp \left(-\sqrt{2 b} \frac{s}{\ell_{p}}\right)
$$

whereas for the bond-director field one obtains

$$
\langle\mathbf{n}(s) \cdot \mathbf{n}(0)\rangle=\frac{d \ell_{p}^{2}}{2 a^{2} \sqrt{b^{2}-c}}\left(\frac{\exp \left(-\left(b-\sqrt{b^{2}-c}\right)^{1 / 2} \frac{s}{\ell_{p}}\right)}{\left(b-\sqrt{b^{2}-c}\right)^{1 / 2}}-\frac{\exp \left(-\left(b+\sqrt{b^{2}-c}\right)^{1 / 2} \frac{s}{\ell_{p}}\right)}{\left(b+\sqrt{b^{2}-c}\right)^{1 / 2}}\right)
$$


The tangent-tangent correlation (Eq.(3i)) is exactly what we obtain for a single worm-like chain, and implies uniform behavior for all temperatures. Eq.(4) on the other hand, indicates a change of behavior at $b^{2}=c$ for the bond-director correlation. The correlation is overdamped for $b^{2}>c$ (high temperatures), while it is underdamped (oscillatory) for $b^{2}<c$ (low temperatures). The interesting point $b^{2}=c$ happens for $u_{c}=$ $2^{19 / 4} /(2+\sqrt{2})^{3 / 2} d \simeq 4.27 / d$, that leads to the value for $T_{c}$ quoted above (see Fig. 1). We also find a divergence in the specific heat, $C_{V}=\frac{\partial^{2} F}{\partial T^{2}}$ where $F=-k_{B} T \log Z$ at $T_{c}$. It should be noted that it is not a thermodynamic phase transition in the sense of long-range ordering and broken symmetry. It is a cross-over that appears due to competing effects, and the transition is from a state with some short-range order to a state with a different shortrange order. Similar phenomena have been observed in Ising-like spin system with competing interactions [7] and the cross-over (transition) point corresponds to a type of 'Lifshitz point' for a 1-d system.

The nature of competition in our double-stranded polymer system can be understood using a plaquette model. Consider rectangular plaquettes attached to each other to form a ribbon. The attached sides (edges) correspond to the bond-director field, while the other sides determine the tangent-director field. The twist degree of freedom corresponds to twisting the plaquettes against each other. The energy expression corresponding to bends comes from the product of the tangent-directors of the neighbouring plaquettes, and has no competition. On the other hand, the energy for twists that comes from the product of the bond-director fields of the neighboring plaquettes, does show competition. These competing effects come from an effective interaction between bonddirectors that are next nearest neighbors. This is due to the fact that alike twists meeting at an edge tend to unwind (annihilate) each other, while unlike twists when they meet are trapped; they do not annihilate each other. This competition is only present at nonzero temperatures and is merely due to topological constraints of the ribbon.

It is useful to study the bond-director correlation in the limiting case $b^{2} \ll c$, that corresponds to relatively low temperatures. Using the asymptotic forms for $b$ and $c$, one obtains $\langle\mathbf{n}(s) \cdot \mathbf{n}(0)\rangle=$ $\sqrt{2} \exp \left(-\left(\frac{d}{4 \ell_{p} a^{2}}\right)^{1 / 3} s\right) \sin \left(\left(\frac{d}{4 \ell_{p} a^{2}}\right)^{1 / 3} s+\frac{\pi}{4}\right)$, for very low temperatures. From the above expressions for the correlation functions, one can read off the persistence lengths $\tilde{\ell}_{\mathrm{TP}}$ and $\tilde{\ell}_{\mathrm{BP}}$, and the pitch $H$, as summarized above.

An intriguing feature of the behaviour of this model is that, although the ground state $(T=0)$ configuration of the system is a flat ribbon, and supports no twists, upon raising the temperature, a twisted structure with short range order emerges. We have confirmed this by perform- ing extensive Molecular Dynamics(MD)/Monte Carlo (MC) simulations of double-stranded semi-flexible polymers. A bead-spring model with bending and stretching energies was used. We combined a velocity-Verlet MD coupled to a heat bath with an off-lattice pivot MC algorithm. The MD was useful for equilibriating the shorter length-scales and MC the long length-scales. Details of the algorithm and computational method will be presented elsewhere [8].

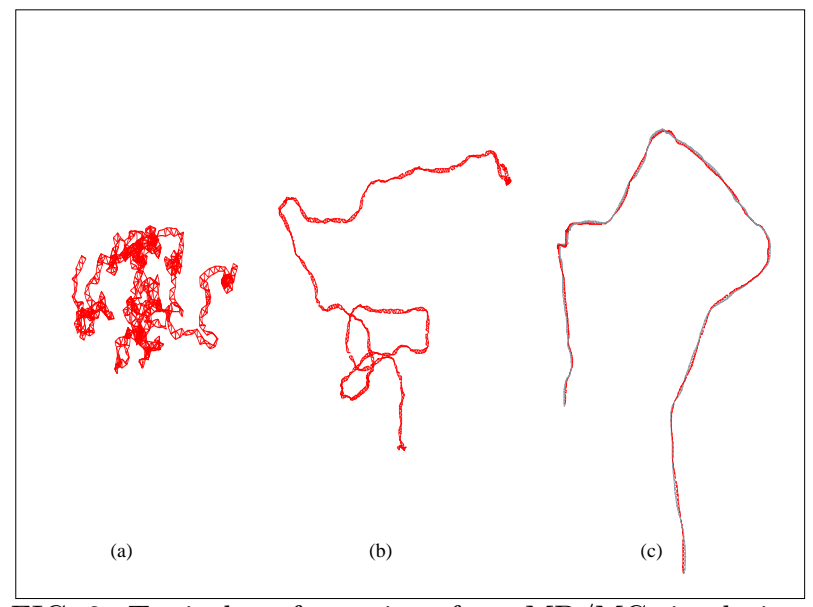

FIG. 2. Typical conformations from MD/MC simulations of a ribbon made up of two chains of 400 monomers (a) above (b) near and (c) below $T_{c}$

We show typical equilibriated conformations in Fig. 2. The snapshots of the polymer configurations suggest that at low temperatures the polymer can be viewed as a collection of long, twisted (straight) rods that are connected by short, highly curved sections of chain which we call "kinks". This picture can be accounted for using a simple argument. We can model our system of two semiflexible polymers subject to the constraint of constant separation, as a semiflexible ribbon, i.e. a semiflexible linear object with anisotropic rigidities whose Hamiltonian reads

$$
\mathcal{H}=\frac{1}{2} \int \mathrm{d} s \sum_{i, j} \kappa_{i j}\left(\frac{\mathrm{d} \mathbf{t}}{\mathrm{d} s}\right)_{i}\left(\frac{\mathrm{d} \mathbf{t}}{\mathrm{d} s}\right)_{j},
$$

where $\kappa_{i j}=\kappa_{\|} n_{i} n_{j}+\kappa_{\perp}\left(\delta_{i j}-t_{i} t_{j}-n_{i} n_{j}\right)$ determines the rigidity anisotropy of the ribbon, corresponding to bending parallel or perpendicular to the bond-director field. The ribbon structure would require $\kappa_{\|} \gg \kappa_{\perp}$. To be consistent with the hard constraint (see above) of constant separation of the polymers, we should take the limit of infinite $\kappa_{\|}$. The Boltzman weight with the above Hamiltonian in the $\kappa_{\|} \rightarrow \infty$ limit can be easily shown to require the constraint $\frac{\mathrm{d} \mathbf{t}(s)}{\mathrm{d} s} \cdot \mathbf{n}(s)=0$, to hold exactly at every point of the ribbon. Recalling $\mathrm{d} \mathbf{t} / \mathrm{d} s=H(s)$ e from the Frenet-Seret equations, where $H(s)$ is the curvature at each point and $\mathbf{e}$ is a unit normal vector to the curve, we can write the constraint as $H(s) \mathbf{e}(s) \cdot \mathbf{n}(s)=0$. 
It tells us that at each point either $H(s)=0$, that is we have straight, twisted sections with no curvature, or $\mathbf{e}(s) \cdot \mathbf{n}(s)=0$, curved regions where the the bonddirector is perpendicular to the curve normal. The former case would correspond to the rod segments whereas the latter would correspond to the "kinks". Performing the configuration sum we obtain that the average separation of the kinks is equal to the tangent-persistence length, which is quite large at small enough temperatures. As the temperature is raised, the kinks get closer so that at some temperature the average kink separation becomes comparable to the size of the kinks, where the rod-kink pattern disappears. This analysis can be understood in the context of the mean-field $(\mathbf{n}, \mathbf{t})$ model above by observing that at low temperatures $\tilde{\ell}_{\mathrm{BP}} \ll \tilde{\ell}_{\mathrm{TP}}$, one can imagine that there are roughly speaking rod-like segments of length $\tilde{\ell}_{\mathrm{TP}}$, each supporting a number of shorter segments of length $\tilde{\ell}_{\mathrm{BP}}$ that are twisted, but de-correlated with one other. As the temperature is raised, the number of twisted rods in each segment $N=\tilde{\ell}_{\mathrm{TP}} / \tilde{\ell}_{\mathrm{BP}}$ decreases very quickly until it saturates to unity at $T=T_{c}$. For higher temperatures the mechanism changes, and the bond correlations are cut off by the tangent fluctuations. Hence, the short-range twist order does not exist anymore. All the main features of the above picture have been observed in the simulation. We plot the $\langle\mathbf{n}(s) \cdot \mathbf{n}(0)\rangle$ correlation function from the simulation in Fig. 3.

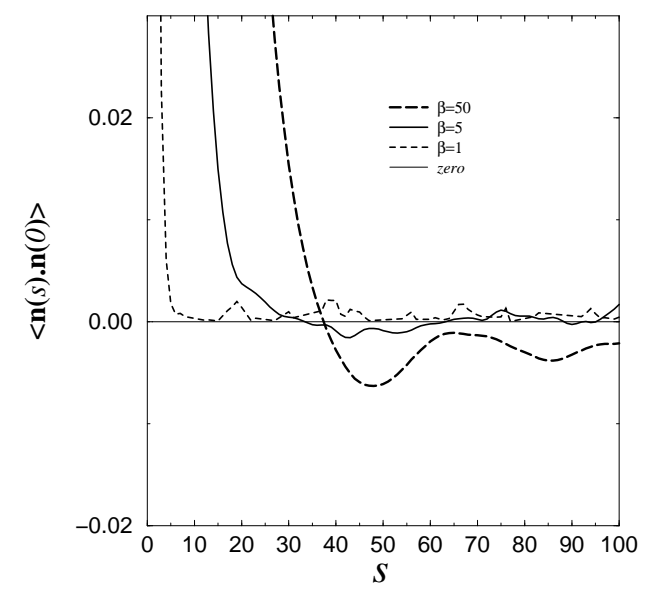

FIG. 3. The $\langle\mathbf{n}(s) \cdot \mathbf{n}(0)\rangle$ correlation function measured in the simulations for temperatures $\beta=1 / k_{B} T=1,5,50$ corresponding to $b^{2}>c, b^{2} \approx c$ and $b^{2}<c$. The averages were done over $\sim 10^{4}$ statistically independent samples.

In conclusion, we have studied the properties of a welldefined model of a double-stranded semi-flexible polymer using a mean-field analytical approach as well as exten- sive MD/MC simulations. We have shown novel nontrivial differences between the high, low and zero temperature behaviour. An alternative formulation of the problem (see Eq.(5) and the following paragraph) where the constraints are implemented in a different way also suggests qualitatively similar results [8]. We also note that the low temperature phase can be used to explain some recent experiments done on single actin filaments by Käs et al [9] who observed a length-scale dependent bending rigidity as well as the kink-rod structure explained above. The high- $q$ small bending rigidity can be associated with the kinks and the low- $q$ large bending rigidity with the rods. Of course, upon averaging the object would have worm-like chain statistics. Finally we mention that there are lots of interesting prospects for the study of such complex linear objects. It is known that biopolymers, such as microtubules, can also be multi-stranded objects. This method could be easily be extended to describe triplestranded objects. The effect of an intrinsic twist changes the ground state but does not change any of the conclusions of our description [8] though we expect it to make the effective persistence length much higher.

We have benefited from numerous discussions with $\mathrm{R}$. Bundschuh, R. Ejtehadi, R Evaerers, E. Frey, G. Grest, M. Kardar and M. Pütz. We would like to thank the hospitality of ICTP, Trieste, where some of this work was done.

[1] O. Kratky and G. Porod, Rec. Trav. Chim., 68, 1106 (1949) .

[2] J.F. Marko and E.D. Siggia, Macromolecules, 27, 981 (1994); B. Fain, J. Rudnick and S. Ostlund, preprint condmat/9610126, (1996).

[3] R. Everaers, R. Bundschuh, and K. Kremer, Europhys. Lett., 29, 263 (1995).

[4] M.G. Bawendi and K.F. Freed, J. Chem. Phys., 83, 2491 (1985); J.B. Lagowski, J. Noolandi, and B. Nickel, J. Chem. Phys., 95, 1266 (1991); A.M. Gupta, and S.F. Edwards, J. Chem. Phys., 98, 1588 (1993); T.B. Liverpool, and S.F. Edwards, J. Chem. Phys., 103, 6716 (1995).

[5] In Ref. [3] it is shown that, if we impose a soft constraint using an energy term like $(k / 2) \int(\Delta s)^{2}$, we can see that the length $l=\left(\kappa / k a^{2}\right)^{1 / 2}$ determines two different regimes; the interesting one being $L \gg l$ ( $L$ is the length of the chains). Hence, our hard constraint in fact, only restricts us to the case of interest.

[6] F. David, and E. Guitter, Europhys. Lett., 5, 709 (1988).

[7] R.M. Hornreich, R. Liebmann, H.G. Schuster and W Selke, Z. Phys., B 35, 91 (1979).

[8] T.B. Liverpool and K. Kremer, in preparation; R. Golestanian and T.B. Liverpool, in preparation.

[9] J. Käs, H. Strey, M. Bärmann and E. Sackmann, Europhys. Lett., 21, 865 (1993). 